\title{
Use of human intravenous immunoglobulin in lower motor neuron syndromes
}

\author{
C M Ellis, S Leary, J Payan, C Shaw, M Hu, M O'Brien, P N Leigh
}

\begin{abstract}
Objective-To determine whether patients with the clinical phenotype of multifocal motor neuropathy but without the electrophysiological criteria for conduction block would respond to intravenous immunoglobulin (IVIg).

Methods-Ten patients were selected with a slowly progressive, asymmetric, lower motor neuron disorder, and were treated prospectively with IVIg at a dose of $2 \mathrm{~g} / \mathrm{kg}$ over 5 days. All subjects had neurophysiological testing to look for evidence of conduction block before treatment. Muscle strength was assessed by MRC grades and hand held myometry, measuring pinch and grip strength. A $20 \%$ increase in both pinch and grip myometry was considered a positive response.

Results-In no patient was conduction block detected. Four of the 10 patients showed a positive response to IVIg, with the best response occurring in two patients who presented with weakness but without severe muscle wasting. Three of the four responders have continued to receive IVIg for a mean period of 17 months (range 15-24 months), with continued effect. The response to IVIg was not related to the presence of anti-GM1 antiganglioside antibodies, but responders had a selective pattern of muscle weakness and normal ( $>90 \%$ predicted) vital capacity.

Conclusion-The findings suggest that a course of IVIg should be considered in patients with the clinical phenotype of multifocal motor neuropathy but without neurophysiological evidence of conduction block.

(F Neurol Neurosurg Psychiatry 1999;67:15-19)
\end{abstract}

Keywords: intravenous immunoglobulin; lower motor neuron; multifocal motor neuropathy

Human intravenous immunoglobulin (IVIg) is established therapy for multifocal motor neuropathy, characterised by conduction block on electrophysiological studies with or without the presence of antiganglioside antibodies. ${ }^{1-3}$ Criteria for defining conduction block have been employed to prevent the misinterpretation of pseudoconduction block (phase cancellation), which can occur in amyotrophic lateral sclerosis. ${ }^{45}$ However, these strict criteria may lead to the misdiagnosis of a potentially treatable condition. ${ }^{6}$ The objective of this study was to determine whether patients with the clinical phenotype of multifocal motor neu- ropathy, but without the electrophysiological criteria for conduction block, would respond to IVIg.

\section{Methods}

We examined prospectively the effect of IVIg in 10 patients attending the motor neuron disease care and research clinic at King's Healthcare. Patient selection was based on a slowly progressive, asymmetric lower motor neuron disorder without bulbar signs or symptoms. Patients with definite upper motor neuron features were excluded, but the preservation of reflexes in an affected limb was not considered an exclusion criterion. The patient details are given in table 1 . Six men and four women were studied. The mean age of onset was 44.7 (SD 12.3) years (range 27-62 years) and the mean duration of disease was 69.8 (SD 45.9) months (range 12-129 months). The onset was in the upper limbs in nine patients and in the lower limbs in one. All patients were tested for IgM anti-GM1 antibodies.

Electrophysiological studies were carried out in all patients by the same investigator (JP). Conventional surface stimulating and recording techniques were employed, and in each patient evidence of conduction block in the nerve supply to small hand and foot muscles was sought, and weak muscles were sampled electromyographically. The criteria of Lange et $a l$ and Katz et al were used to define conduction block. ${ }^{5}{ }^{6}$ Thus the CMAP amplitude and area must fall by at least $50 \%$ whereas the CMAP duration must not increase by more than $30 \%$. However, the exhaustive nerve conduction studies described by Lange et al and by Katz et al were not performed. ${ }^{56}$ Thus clinically normal muscles were not always investigated, nerve roots were not stimulated, and motor conduction velocities were not always measured when the corresponding $\mathrm{F}$ wave was of normal or slightly increased latency.

Patients were admitted to hospital and treated with IVIg at a dose of $2 \mathrm{~g} / \mathrm{kg}$ over a period of 5 days. Clinical assessment was carried out by the same examiner before and 10-14 days after treatment in all patients. Muscle strength was assessed using Medical Research Council (MRC) gradings in 28 muscle groups and hand held myometry, assessing pinch (Pinch gauge, B and L Engineering, Sante Fe Springs, CA, USA) and grip strength (JAMAR 5030J1 hand dynamometer, Sammons Preston, Jackson, MI, USA). Each patient had three attempts at the hand held myometry, and the maximum value was recorded. A $20 \%$ improvement in both pinch 
Table 1 Patient details, summary of response and final diagnosis at follow up

\begin{tabular}{|c|c|c|c|c|c|c|c|c|c|c|c|c|c|}
\hline \multirow[b]{2}{*}{ Subject } & \multirow[b]{2}{*}{ Sex } & \multicolumn{3}{|l|}{ Onset } & \multirow[b]{2}{*}{$\begin{array}{l}\text { Age of } \\
\text { onset }(y)\end{array}$} & \multirow[b]{2}{*}{$\begin{array}{l}\text { Duration } \\
\text { (months) }\end{array}$} & \multirow[b]{2}{*}{$\begin{array}{l}F V C \\
(\%)\end{array}$} & \multirow[b]{2}{*}{$A b$} & \multirow[b]{2}{*}{ Fasc } & \multirow[b]{2}{*}{ Atrophy } & \multirow[b]{2}{*}{ Cramps } & \multirow[b]{2}{*}{ Reflexes } & \multirow[b]{2}{*}{$\begin{array}{l}\text { Diagnosis at } \\
\text { follow up }\end{array}$} \\
\hline & & $\begin{array}{l}\text { Proximal/ } \\
\text { distal }\end{array}$ & $\begin{array}{l}\text { Upper/lower } \\
\text { limb }\end{array}$ & $\begin{array}{l}\text { Unilateral/ } \\
\text { bilateral }\end{array}$ & & & & & & & & & \\
\hline 1 & $\mathrm{~F}$ & Distal & Lower & Unilateral & 60 & 120 & 83 & $\mathrm{Neg}$ & No & Yes & Yes & $\downarrow$ & SMA \\
\hline 2 & M & Distal & Upper & Unilateral & 35 & 94 & 82 & $\mathrm{Neg}$ & Yes & Yes & No & $\mathrm{N}$ & ALS \\
\hline 3 & $M$ & Proximal & Upper & Bilateral & 48 & 93 & 84 & Pos & Yes & Yes & Yes & $\downarrow$ & ALS \\
\hline 4 & $M$ & Distal & Upper & Unilateral & 45 & 129 & 92 & Pos & No & Selective & Yes & $\downarrow$ & $M M N$ \\
\hline 5 & $M$ & Distal & Upper & Bilateral & 58 & 27 & 84 & Neg & Yes & Yes & Yes & $\downarrow$ & ALS \\
\hline 6 & $\mathrm{~F}$ & Distal & Upper & Unilateral & 37 & 20 & 100 & $\mathrm{Neg}$ & No & No & Yes & $\downarrow$ & $M M N$ \\
\hline 7 & $M$ & Distal & Upper & Unilateral & 62 & 17 & 93 & $\mathrm{Neg}$ & Yes & Yes & Yes & $\downarrow$ & ALS \\
\hline 8 & $\mathrm{~F}$ & Distal & Upper & Bilateral & 32 & 105 & 102 & Neg & No & Yes & No & $\mathrm{N}$ & MMN \\
\hline 9 & $\mathrm{~F}$ & Distal & Upper & Unilateral & 27 & 12 & 101 & $\mathrm{Neg}$ & No & Yes & No & $\downarrow$ & MMN? \\
\hline 10 & $M$ & Distal & Upper & Unilateral & 43 & 81 & NK & $\mathrm{Neg}$ & No & Selective & no & $\downarrow$ & $M M N$ \\
\hline
\end{tabular}

Duration=duration of disease; $\mathrm{FVC}=$ forced vital capacity; $\mathrm{Ab}=$ antiganglioside antibodies; Fasc $=$ fasciculations, $\mathrm{N}=$ normal, $\downarrow=$ reduced; selective atrophy indicates selective involvement of individual muscles within a myotome; $\mathrm{NK}=$ not known; $\mathrm{SMA}=$ spinal muscular atrophy; $\mathrm{ALS}=\mathrm{amyotrophic}$ lateral sclerosis; $\mathrm{MMN}=$ multifocal motor neuropathy;

and grip myometry was considered a positive response.

When possible, treatment was repeated after a period of $4-10$ weeks.

\section{Results}

NEUROPHYSIOLOGY

When present, $F$ waves were of normal or marginally increased latency. In one patient (patient 10) foot muscle $\mathrm{F}$ waves were absent, but the $M$ wave amplitudes were less than 200 $\mu \mathrm{V}$. In patients 2 and 7 the thenar $\mathrm{F}$ wave was absent on one side. Patient 2 showed a normal evoked thenar compound muscle action potential with no decrement on stimulation up to the axilla. In patient 7 , the evoked thenar potential was of moderately reduced amplitude and its $\mathrm{F}$ wave was absent. On the contralateral side, the thenar $\mathrm{F}$ was normal but the hypothenar $\mathrm{F}$ latency was $36 \mathrm{~ms}$ and the distally evoked hypothenar potential of $0.85 \mathrm{mV}$ showed a $41 \%$ decrement, with no change in form on stimulating above the elbow.

In one patient (8) evoked hypothenar muscle potentials were of normal amplitude and $\mathrm{F}$ wave latency, yet the muscles were weak and wasted. A second such discrepancy was found in patient 6 , in whom a weak abductor pollicis brevis and first dorsal interosseous showed no EMG evidence of denervation or reinnervation and evoked thenar and hypothenar muscle action potentials were of normal amplitude and $\mathrm{F}$ wave latency.

Sural nerve sensory action potentials were normal in eight patients and markedly reduced in one (5), in whom the median digital potential was also small. Sural potentials were not sought in one patient (8) in whom clinical abnormality was confined to the upper limbs and sensory potentials were normal. In patient 4 , radial, median, and ulnar sensory potentials on the side first affected by weakness had only half the amplitude of the normal potentials on the more recently affected arm. This was also true of the radial nerve potential in patient 10 .

IMMUNOGLOBULIN TREATMENT

The results of the MRC grades and dynamometry before and 10-14 days after the first course of IVIg treatment are displayed in table 2. The percentage changes are shown in table 3 . Four of the 10 patients $(4,6,8$, and 10) showed a response to IVIg as determined by a $>20 \%$ increase in myometry measures of both pinch and grip in an affected limb. We thought that the MRC grades, which are non-linear, were a poor indicator of clinical response and too subjective to use as an outcome measure. Using the paired $t$ test, there was no significant difference in MRC grading pretreatment and post-treatment in the responders $(p=0.16)$.

There was no correlation between the response to IVIg and the age of onset of weakness, duration of disease, or sex of patient $(p>0.15)$. The presence of antiganglioside antibodies did not determine response. The forced vital capacity, measured before treatment, was positively correlated with the change in grip dynamometry $(\mathrm{p}=0.024)$, but failed to reach significance with pinch measures $(\mathrm{p}=0.19)$.

Patient 9 did not show any appreciable change in myometry or MRC grades used in our protocol, but described a definite functional improvement. She has therefore contin-

Table 2 MRC gradings in 14 muscle groups; pinch and grip myometry values (kg) pretreatment and 14 days post-IVIg therapy

\begin{tabular}{|c|c|c|c|c|c|c|c|c|c|c|c|c|}
\hline \multirow[b]{2}{*}{ Patient } & \multicolumn{2}{|c|}{ Right $M R C$ (/70) } & \multicolumn{2}{|c|}{ Right pinch (kg) } & \multicolumn{2}{|c|}{ Right grip (kg) } & \multicolumn{2}{|c|}{ Left $M R C(/ 70)$} & \multicolumn{2}{|c|}{ Left pinch $(\mathrm{kg})$} & \multicolumn{2}{|c|}{ Left grip (kg) } \\
\hline & Pre & Post & Pre & Post & Pre & Post & Pre & Post & Pre & Post & Pre & Post \\
\hline 1 & 44.5 & 46.5 & 1.0 & 1.5 & 17.0 & 18.0 & 42.5 & 45.0 & 1.0 & 1.5 & 14.0 & 10.0 \\
\hline 2 & 67.0 & 66.5 & 9.5 & 10.5 & 32.0 & 30.0 & 62.0 & 63.5 & 11.0 & 11.0 & 44.0 & 46.0 \\
\hline 3 & 54.0 & 59.0 & 3.5 & 4.0 & 22.0 & 20.0 & 62.0 & 66.0 & 7.5 & 7.2 & 38.0 & 35.0 \\
\hline 4 & 62.5 & 62.5 & 8.0 & 13.0 & 25.0 & 30.0 & 61.0 & 68.0 & 1.0 & 1.0 & 22.0 & 23.0 \\
\hline 5 & 63.5 & 65.5 & 4.0 & 4.0 & 20.0 & 20.0 & 65.5 & 66.0 & 4.0 & 4.0 & 22.0 & 18.5 \\
\hline 6 & 62.5 & 67.5 & 6.5 & 8.75 & 22.0 & 31.5 & 70.0 & 70.0 & 9.2 & 8.0 & 28.0 & 24.0 \\
\hline 7 & 67.5 & 68.0 & 3.0 & 3.0 & 24.0 & 24.5 & 69.5 & 70.0 & 9.0 & 7.5 & 32.0 & 36.0 \\
\hline 8 & 61.5 & 61.0 & 2.0 & 1.0 & 19.0 & 24.0 & 60.0 & 60.5 & 1.0 & 3.0 & 12.0 & 24.0 \\
\hline 9 & 62.0 & 62.0 & 3.0 & 2.5 & 10.5 & 12.0 & 61.5 & 63.5 & 4.5 & 5.0 & 16.0 & 17.0 \\
\hline 10 & 70.0 & 70.0 & 14.5 & 14.0 & 43.0 & 50.0 & 60.0 & 66.5 & 7.0 & 12.5 & 30.0 & 52.0 \\
\hline
\end{tabular}

Objective responders are in bold. 


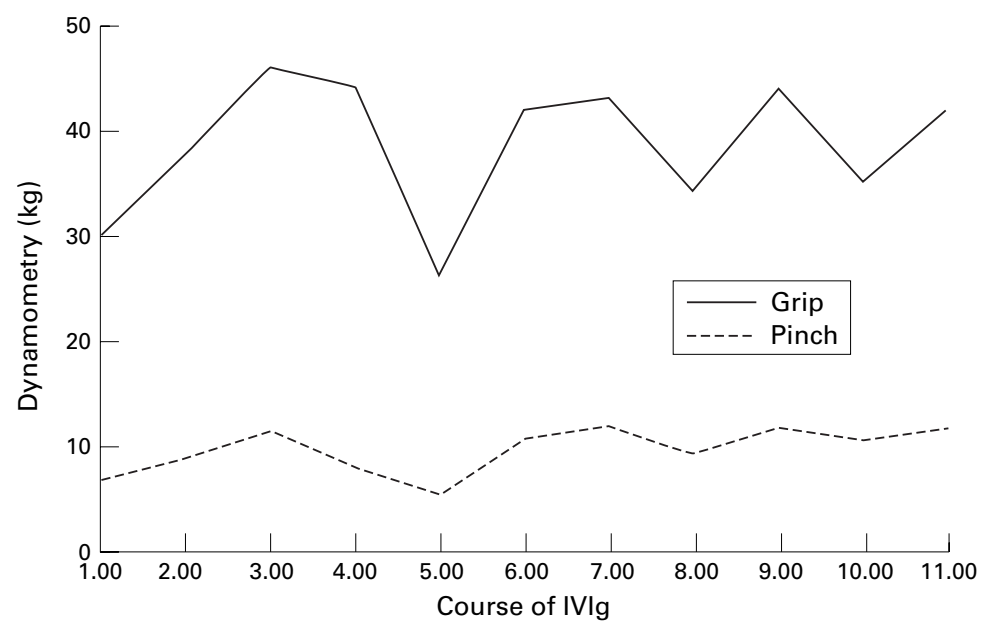

Values for pinch and grip dynamometry (kg) before treatment in 11 successive courses of IVIg in patient 10.

ued to receive treatment, but despite four courses, has shown no objective improvement.

\section{Adverse effects}

Mild side effects of headache, nausea, or fever occurred during administration of the IVIg in seven of the 10 patients. Patient 7 developed pallor, sweating, and hypotension within a few minutes of administration of the second course of IVIg and therefore treatment was stopped.

Patient 5 developed an erythrodermal skin rash 9 days after completion of the first course of IVIg. Despite oral steroids, this progressed requiring admission to hospital for supportive treatment. He was thought to have late onset atopic eczema, aggravated by the immunotherapy. The rash has continued, and at 6 months, the dermatologists have introduced azathioprine.

\section{Follow up}

The mean follow up period has been 18 (SD 3.2) months (range 14-24 months). Follow up has been assessed by repeated pinch and grip measures and MRC grading of muscle strength during clinical examination, and by the functional reports of the patients.

\section{Responders}

Patient 4 has received treatment for 15 months, and after an initial improvement, seems to have stabilised with no further documented improvement in muscle strength, as measured by

Table 3 Percentage change in MRC rating and pinch and grip myometry measures 14 days post-treatment with IVIg

\begin{tabular}{lllllll}
\hline Patient & \multirow{2}{*}{ Right MRC } & Right pinch & Right grip & Left MRC & Left pinch & Left grip \\
\hline $1 \ddagger$ & 4.5 & 50.0 & 5.9 & 5.9 & 50.0 & -28.6 \\
$2 \ddagger$ & -0.8 & 10.5 & -6.3 & 2.4 & 0.0 & 4.6 \\
$3 \dagger$ & 9.3 & 14.3 & -9.1 & 6.5 & -4.0 & -7.9 \\
$\mathbf{4}^{\star}$ & $\mathbf{0 . 0}$ & $\mathbf{6 2 . 5}$ & $\mathbf{2 0 . 0}$ & $\mathbf{1 1 . 5}$ & $\mathbf{0 . 0}$ & $\mathbf{4 . 6}$ \\
$5 \ddagger$ & 3.2 & 0.0 & 0.0 & 0.8 & 0.0 & -15.9 \\
$\mathbf{6}^{\star}$ & $\mathbf{8 . 0}$ & $\mathbf{3 4 . 6}$ & $\mathbf{4 3 . 2}$ & $\mathbf{0 . 0}$ & $\mathbf{- 1 3 . 0}$ & $\mathbf{- 1 4 . 3}$ \\
$7 \ddagger$ & 0.7 & 0.0 & 2.1 & 0.7 & -16.7 & 12.5 \\
$\mathbf{8 \dagger}$ & $\mathbf{- 0 . 8}$ & $\mathbf{- 5 0 . 0}$ & $\mathbf{2 6 . 3}$ & $\mathbf{0 . 8}$ & $\mathbf{2 0 0 . 0}$ & $\mathbf{1 0 0 . 0}$ \\
$9^{\star}$ & 0.0 & -16.7 & $\mathbf{1 4 . 3}$ & 3.3 & 11.1 & 6.3 \\
$\mathbf{1 0}$ & $\mathbf{0 . 0}$ & $\mathbf{- 3 . 5}$ & $\mathbf{1 6 . 3}$ & $\mathbf{1 0 . 8}$ & $\mathbf{7 8 . 6}$ & $\mathbf{7 3 . 3}$ \\
\hline
\end{tabular}

«Subjective report of improvement.

tSubjectively unsure whether improvement has occurred.

$\ddagger$ No improvement subjectively.

Objective responders in bold. repeated pinch and grip and MRC grades. His functional improvement particularly allowed him to write more easily, which was important as he was self employed, and we therefore thought that this warranted continued treatment. We now plan to monitor his strength to determine whether further courses of IVIg are needed.

Patient 6 has been treated with $2 \mathrm{~g} / \mathrm{kg}$ IVIg for 22 months, at 2 monthly intervals. With this regime, her strength remains constant. Her functional response was dramatic, enabling her to use her right hand "instinctively" for the first time in many months. For example, her power of finger extension on the right was MRC grade 2 before treatment and grade 4 after treatment. She rates her functional improvement to $60 \%$ normal as judged by weight training performance. A dose of $1 \mathrm{~g} / \mathrm{kg}$ IVIg led to a good initial response but only lasting for 1 month, and an interval of 2.5 months led to increasing weakness, which responded more slowly to treatment.

Patient 10 has received $2 \mathrm{~g} / \mathrm{kg}$ IVIg at $4-6$ weekly intervals for 24 months to date, with continuing efficacy. Functionally, his walking has improved such that he can wade in the river while fishing and run a short distance. He can now use his computer without an arm rest, button shirts, clean his teeth, and drive a car. Once again, a trial of $1 \mathrm{~g} / \mathrm{kg}$ led to a good response, but lasted a shorter time. To investigate whether treatment affects the overall progression of the condition, the pretreatment dynamometry values for patient 10 are displayed in the figure.

Although patient 8 had an objective response to the IVIg, she was not convinced that there was a functional improvement, and therefore she decided not to continue with the treatment after the second course.

\section{Non-responders}

Patients 3, 5, and 7 (follow up 18, 18, and 19 months) have developed definite upper motor neuron signs and fulfil El Escorial diagnostic criteria for clinically probable (5 and 7) and possible (3) motor neuron disease. Patient 1 remains stable (follow up 18 months), and is considered to have progressive spinal muscular atrophy. Patient 2 (follow up 18 months) has developed upper and lower motor neuron signs, but has an atypical course with long periods of stability followed by decline, and is considered to have atypical motor neuron disease. Patient 9 may have a spinal muscular atrophy, or may have simply responded too poorly to the IVIg to detect an objective response. The non-responders who have now been classified as amyotrophic lateral sclerosis notably had fasciculations at presentation.

\section{Discussion}

We have shown a response to IVIg in four out of 10 patients presenting with a progressive lower motor neuron disorder, clinically impossible to distinguish with certainty from multifocal motor neuropathy. Intensive electrophysiological study of such patients led Lange et al to the conclusion that, whereas conduction ab- 
normalities can be an incidental finding in some patients with multifocal motor neuropathy, there may be a distinct syndrome characterised by the presence of conduction block in two or more nerves. ${ }^{5}$

Our study was not designed as an electrophysiological study, rather as a pragmatic clinical approach to the problem of lower motor neuron syndromes which might or might not respond to IVIg. Therefore, the exhaustive nerve conduction studies described by Lange et al and by Katz et al were not performed. ${ }^{5}$ It should be recalled that Lange et al found absence or extreme delay of $\mathrm{F}$ waves whenever there was conduction block. We did not find conduction block with the methods used in our clinic. In two of our patients ( 2 and 7 ), the thenar $\mathrm{F}$ wave was absent, and it is possible that conduction block of median motor fibres might have been disclosed by stimulation at the spinal cord level in these patients. Neither patient benefited from treatment.

Two of the four patients who responded to IVIg (6 and 8) did show precisely what might have been expected with conduction blocknamely, weakness in muscles without wasting, with normal evoked compound muscle action potential amplitudes. However, F wave latencies were normal in both.

The significance, if any, of the sensory potential asymmetries in two of the patients who responded to treatment (4 and 10) is unknown.

The response of two of our patients ( 6 and 10) has been indisputable, and contrary to the recent report by Wokke et $a l^{7}$ the effect has been maintained on repeated treatments for 17 and 19 months respectively. Neither of our patients had significant atrophy at presentation, and their excellent response to treatment is consistent with the findings of Bouche et al, who divided their patients with multifocal motor neuropathy into those with pronounced amyotrophy and those without. ${ }^{2}$ The second group had an initial and sustained improvement with IVIg, but the group with amyotrophy responded less well, and the response was not sustained.

Azulay et al described the long term treatment of IVIg in a series of 18 patients followed up for a mean of 25.3 months. ${ }^{8}$ The mean duration of action of IVIg was 53 days. The half life of immunoglobulin in the serum is 3-4 weeks, and it is possible that patients relapse when the serum concentration of immunoglobulin falls below a critical level. This level is likely to be variable between patients, and possibly even within an individual patient, and would explain why infusions at varying time intervals are required to maintain the clinical effect. The maintenance dose of IVIg has varied in different studies, and the optimal dose is again likely to be variable in individual patients. The total serum IgG concentration shows no correlation with clinical response, even within the same patient, ${ }^{9}$ and therefore cannot be used to determine the dose of IVIg in an individual patient. To date, we have delivered $2 \mathrm{~g} / \mathrm{kg}$ as a maintenance dose in patients 6 and 10, at 8 and 6 week intervals, as attempts to reduce this have resulted in a shorter duration of efficacy. This is a time consuming and costly treatment, and the functional benefits to the patient must outweigh the inconvenience.

The effect of IVIg on disease progression is not clear. The pretreatment values for pinch and grip displayed in the figure seem to be relatively stable, but it is interesting that the interval between the 4 th and 5 th courses was extended to 8 weeks, and the values for pinch and grip are both below baseline concentrations before IVIg was started. This may suggest that the disease process is progressing but strength is maintained by the IVIg.

$\operatorname{IgM}$ anti-GM1 antibodies are reported in $50 \%-60 \%$ of cases of multifocal motor neuropathy, ${ }^{2}{ }^{8}$ and have usually not predicted response to immunoglobulin treatment, ${ }^{10}$ consistent with our own findings. However, this is controversial, and Azulay et al found that 11 of 12 patients who responded to IVIg had high titres of anti-GM1 antibodies, and a correlation existed between the initial presence of high titres of antibodies and a long lasting response to IVIg. ${ }^{8}$ The antibody concentration did not change with treatment. The discrepancy may in part relate to the methodology used in the measurement, ${ }^{11}$ and Pestronk et al have reported a sensitivity of anti-GM1 testing of $85 \%$ for multifocal motor neuropathy. ${ }^{12}$ The duration of disease did not determine response to treatment, as previously described in multifocal motor neuropathy.

The identification of treatment response has been considered previously. ${ }^{13}$ Myometry readings tend to be reliable for a single investigator, and are capable of detecting small changes in muscle strength. ${ }^{14}{ }^{15}$ The MRC scale has proved less useful as an objective indicator of treatment response, ${ }^{13}$ as more significant improvements in muscle strength are needed before a change in MRC grading is achieved. In our study, the MRC grades did not differ significantly after IVIg, in keeping with these findings.

Neurophysiological protocols to detect distal and proximal disturbances of motor nerve conduction in the clinical setting may improve identification of multifocal motor neuropathy. Magnetic resonance neurography has been used to directly visualise the proximal portions of cervical spinal nerves to show high signal intensities in cervical radiculopathy, ${ }^{16}$ and nerve root enlargement is reported using MRI in chronic inflammatory demyelinating polyneuropathy. ${ }^{17}$ As these techniques improve, they may allow detection of proximal demyelination to improve the diagnosis of multifocal motor neuropathy.

Our findings suggest that failure to detect conduction block in weak muscles on routine clinical testing (rather than following an exhaustive research protocol) does not exclude a response to IVIg in patients with the clinical phenotype of multifocal motor neuropathy. We were particularly struck at clinical presentation by the selective pattern of muscle weakness in responders. Also, they tended to have normal or near normal vital capacity at presentation. 
Those with little muscle wasting showed the best response in our series, and have maintained clinical and functional benefit over repeated treatments. The lack of muscle wasting is consistent with maintained axonal integrity, which is eventually lost due to motor nerve damage or death. The optimum dosage and time interval between treatments is likely to vary between patients. All patients showed some response after the first course of treatment, and in the follow up series reported by Azulay et al, the maximum benefit was gained by the third treatment. ${ }^{8}$ The questions of when to stop treatment as patients stabilise, and whether to use other immunosuppressants to modify the course of the disease, remain unanswered. Further information on clinical prediction of response to IVIg, and conversely, on prediction of evolution to motor neuron disease (amyotrophic lateral sclerosis) is needed and could best be acquired through multicentre studies, as these patients are relatively rare.

This work was supported by the Motor Neuron Disease Association UK and Action Research.

1 Van den Berg LH, Kerkhoff H, Oey PL, et al. Treatment of multifocal motor neuropathy with high dose intravenous immunoglobulin: a double blind, placebo controlled study. 7 Neurol Neurosurg Psychiatry 1995;59:248-52.

2 Bouche P, Moulonguet A, Younes-Chennoufi AB, et al. Multifocal motor neuropathy with conduction block: a study of 24 patients. $\mathcal{F}$ Neurol Neurosurg Psychiatry 1995;59: $38-44$

3 Van den Berg LH, Franssen H, Wokke JHJ. Improvement of multifocal motor neuropathy during long term weekly treatment with human immunoglobulin. Neurology 1995 45:987-8.
4 Cornblath DR, Asbury AK, Albers JW, et al, for Ad Hoc Subcommittee of the American Academy of Neurology AIDS Task Force. Research criteria for diagnosis of chronic inflammatory demyelinating polyneuropathy (CIDP). Neurology 1991;41:617-18.

5 Lange DJ, Trojaborg W, Latov N, et al. Multifocal motor neuropathy with conduction block: is it a distinct clinical entity? Neurology 1992;42:497-505.

6 Katz JS, Wolfe GI, Bryan WW, et al. Electrophysiological findings in multifocal motor neuropathy. Neurology 1997 ; 48:700-7.

7 Van den Berg LH, Franssen H, Van Doorn PA, et al. Intravenous immunoglobulin treatment in lower motor neuron disease associated with highly raised anti-GM1 antibodies. f Neurol Neurosurg Psychiatry 1997;63:674-7.

8 Azulay J-Ph, Rihet $\mathrm{P}$, Pouget J, et al. Long term follow up of multifocal motor neuropathy with conduction block under treatment. F Neurol Neurosurg Psychiatry 1997;62:391-4.

9 Sewell WAC, Brennan VM, Donaghy M, et al. The use of self infused immunoglobulin home therapy in the treatment of acquired chronic demyelinating neuropathies. $\mathcal{F}$ Neurol Neurosurg Psychiatry 1997;63:106-9.

10 Parry GJ. Antiganglioside antibodies do not necessarily play a role in multifocal motor neuropathy. Muscle Nerve 1994; 17:97-9.

11 Van Schaik IN, Bossuyt PM, Brand A, et al. Diagnostic value of GM1 antibodies in motor neuron disorders and neuropathies: a meta-analysis. Neurology 1995;45:1570-7.

12 Pestronk A, Choksi R, Multifocal motor neuropathy. Serum IgM anti-GM1 ganglioside antibodies in most patients detected using covalent linkage of GM1 to ELISA plates. Neurology 1997;49:1289-92.

13 Thompson N, Choudhary P, Hughes RAC, et al. A novel trial design to study the effect of intravenous immunoglobulin in chronic inflammatory demyelinating polyranoglobulin in chronic inflammatory demyeling

14 Bohannon RW. Test-retest reliability of hand held dynamometry during a single session of strength assessment. Phys Ther 1986;66:206-9.

15 Lennon SM, Ashburn A. Use of myometry in the assessment of neuropathic weakness: testing for reliability in clinical practice. Clin Rehabil 1993;7:125-33.

16 Dailey AT, Tsuruda JS, Goodkin R, et al, Magnetic resonance neurography for cervical radiculopathy: a resonance neurography for cervical radiculop
preliminary report. Neurosurgery 1996;38:488-92.

17 Schady W, Goulding PJ, Lecky BRF, et al. Massive nerve root enlargement in chronic inflammatory demyelinating polyneuropathy. F Neurol Neurosurg Psychiatry 1996;61: $636-40$. 